\title{
Preempting critical care services for patients with hematological malignancies
}

\author{
Élie Azoulay ${ }^{1 *}$ (D, Marcio Soares ${ }^{3}$ and Étienne Lengliné ${ }^{2}$
}

๑ 2021 Springer-Verlag GmbH Germany, part of Springer Nature

An increasing number of critically ill patients is immunocompromised [1,2], and half of them are treated for hematological malignancies [3]. In hematology patients, admission in an intensive care unit (ICU) might be required at different time points throughout the disease trajectory: organ infiltration and compression by aggressive or bulky malignancies [4], tumor lysis syndrome and metabolic complications of diseases with high tumoral burden [5], bacterial or non-bacterial infections [6], drugs, chimeric antigen receptor $\mathrm{T}$ cells (CAR-T) therapy or stem cell transplantation-related toxicity, disease progression, as well as decompensation of associated comorbidities [7]. Even though mortality rate of critically ill patients with hematological malignancies is twice as high compared to non-immunocompromised patients $[7,8]$, critical care services are used as a bridge to cure. For instance, in ICU survivors, response to chemotherapy, remission, quality of life and 1-year survival, are substantial, and not significantly lower than those in patients who did not require ICU admission [7, 9-13].

Little is known on the global burden of critical illness in patients with hematological malignancies. In this issue of the journal, Ferreyro and coworkers provide the results of a population-based cohort study of adults with a new diagnosis of hematologic malignancy in Ontario, Canada (2006-2017) [14]. Large scale evaluation of the incidence and the predictors of ICU admission within 1-year of hematologic malignancy diagnosis was not available until now. Moreover, the study was very well carried out, and data from a large province of almost 15 million populations were extremely well handled. These results are novel and impactful. The finding that the 1-year

\footnotetext{
*Correspondence: elie.azoulay@aphp.fr

${ }^{1}$ Critical Care Department, Saint-Louis Hospital, Paris, France

Full author information is available at the end of the article
}

incidence of ICU admission ranges from 7 to $22 \%$ according to the type of malignancy, associated comorbidities and baseline laboratory abnormalities should invite every hospital decision maker to anticipate the number of ICU beds to be dedicated to patients with hematological malignancies. These valuable data can also serve as a basis for hematologists in charge of patients to provide clear and fair information about the care pathway including ICU as a possible step rather than an unthinkable accident. Moreover, as half of these admissions are needed within 30 days of the diagnosis, it is likely that most patients with aggressive lymphoma or hyperleukocytic leukemia, as well as those presenting with acute respiratory failure, acute kidney injury, neurological, liver or heart involvement from disease infiltration and tumoral burden had been admitted directly to the ICU. Here again, at each institution, based on the level of monitoring, the continuity of medical and nursing care, as well as the use of life-saving therapy outside the ICU (i.e., noninvasive ventilation), newly diagnosed patients with aggressive hematological malignancies should be stratified for their risk of pulmonary leukemic infiltration, leukostasis, tumor lysis syndrome, organ infiltration or compression by a bulky tumor, metabolic complications, etc. [15-18]. Those patients would be in a safer environment in the ICU where they are managed concomitantly by ICU specialists and hematologists. Therefore, based on these results and those from the literature (Fig. 1), every institution should design and organize an ICU whose size, structure, staff, equipment and environment are best adapted to these patient pathways.

This study needs to be replicated in other countries and settings. It is likely that these incidences of ICU admission have been underestimated as triage procedures for ICU admission have selected the best candidates for full critical care management. ICU bed shortages is also often an issue. However, with increasing short- and long-term

\section{Springer}




\section{Baseline cohort}

Acute Myeloid Leukemia

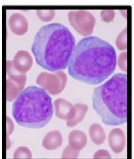

幅幅
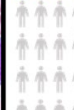

Aggressive NHL

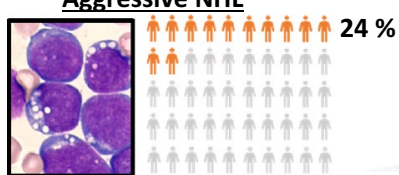

Acute Lymphoblastic Leukemia
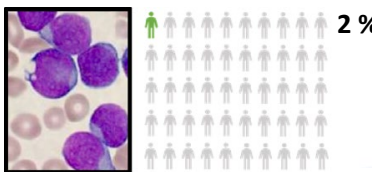

Myelodysplastic Syndromes

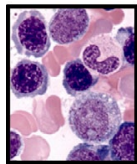

9\%

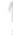

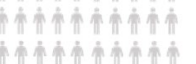

Multiple Myeloma

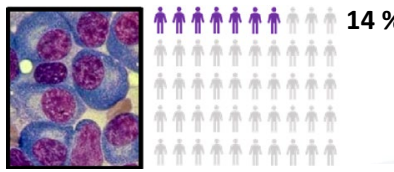

Myeloproliferative Neoplasms

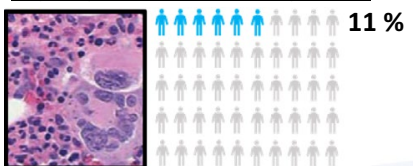

Hodgkin lymphoma

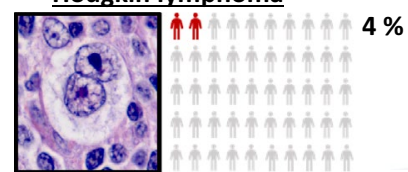

Chronic Lymphoid leukemia

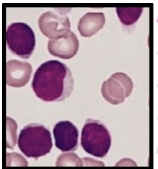

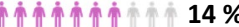

Indolent NHL

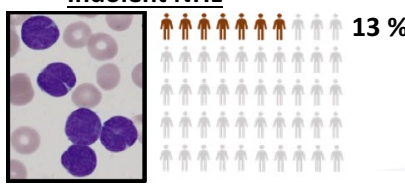

$\%$

$\%$

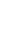

$\%$

\section{Year Rate of ICU \\ admission}
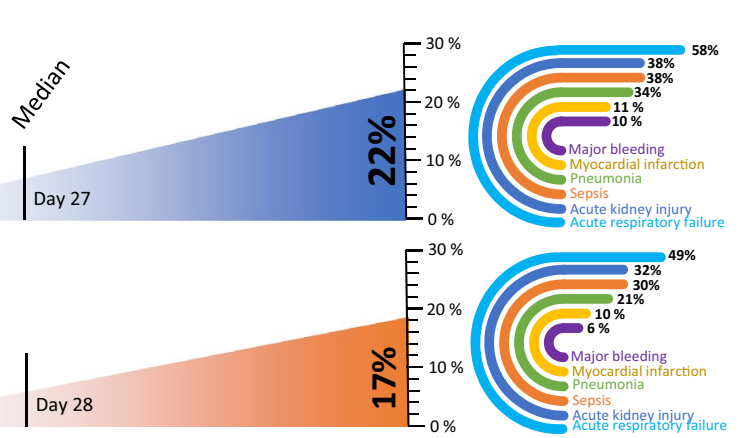

$32 \% 43 \%$

咲启

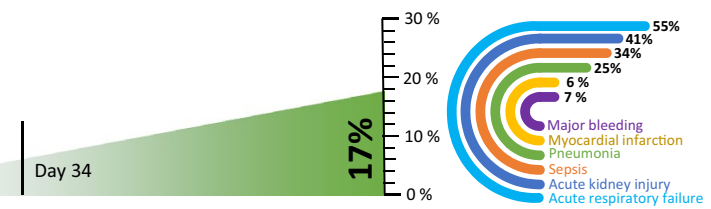

吕茣茣

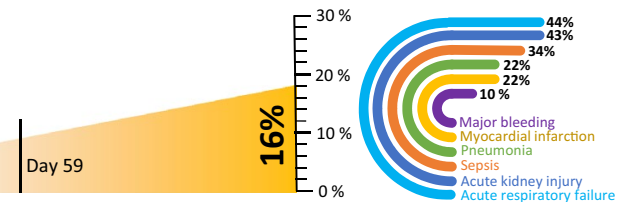

㕉糪
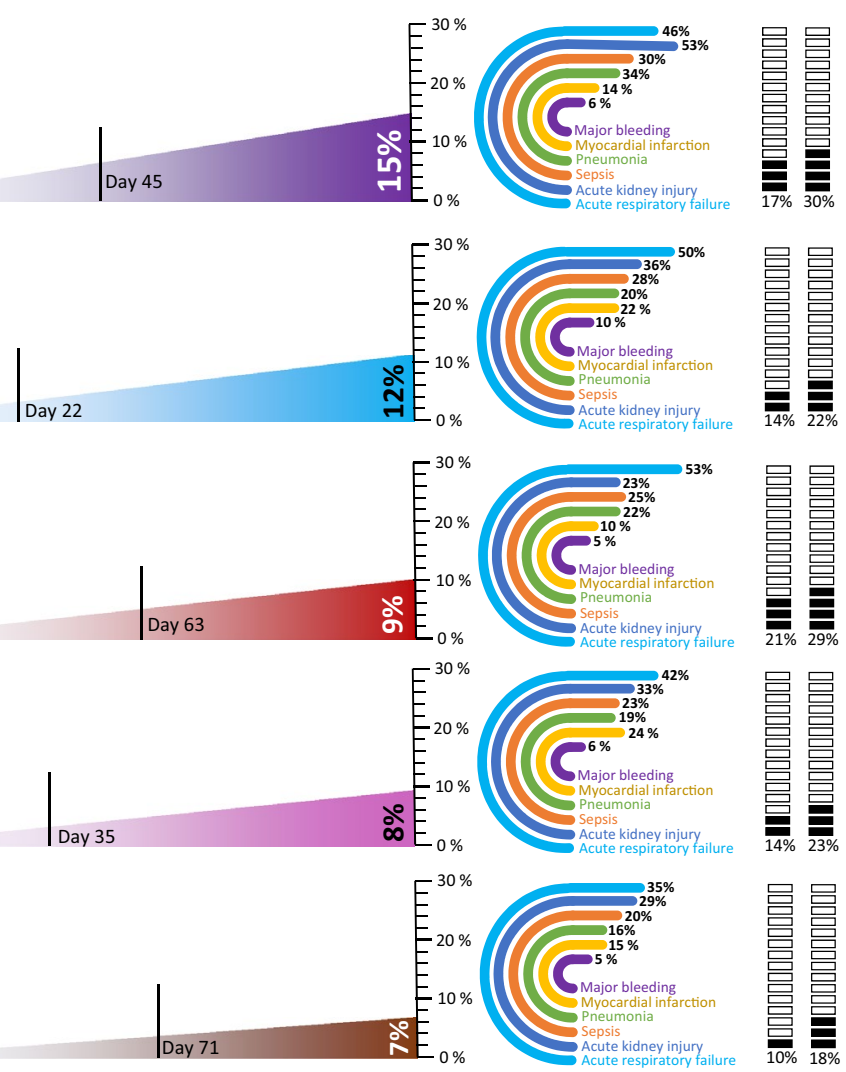

Fig. 1 Baseline cohort vs. 1 Year Rate of ICU admission vs. ICU Conditions Mortality 
survival and complete remission rates reported in critically ill hematology patients [12], clinicians need to open the ICU doors to more patients with malignancies, as long as critical care management does not represent a non-beneficial and aggressive care, and as long as patients consent for undergoing ICU treatments that represent a bridge to prolonged cure. Also, the cumulative incidence curve for admission to the ICU does not seem to show a plateau after one-year, and it will be interesting to know whether there is an increased risk of admission in the longer term in certain subgroups. Another challenging aspect with the management of patients with hematological malignancies is the need for both specialists to understand and respect the other professional competencies. The best outcomes can only result from optimal and fruitful collaboration between ICU specialists and hematologists, in respect of others, and with the deep hope that this musical score played with four hands will translate into improved outcomes. Both specialists should train together to acquire core competencies for the management of hematology patients, and learn together which protocols should be implemented or updated at each place, according to patient's case mix and local peculiarities. Future studies should also correlate outcomes to quantitative and qualitative use of critical care services for hematology patients. Quality indicators depicting appropriate use of intensive treatments should also be monitored to avoid disproportionate care (both excessive or insufficient level of care). While it is non-desirable to provide aggressive care to patients with no life-span expanding therapy, shortening durations or intensity of time-limited trials might hamper optimal outcomes to be obtained. Along this line, delaying life-saving treatments or struggling about starting chemotherapy when it's the only option that offers short- and long-term disease control, quality of life and survival is inappropriate and the source of unwanted outcomes. In keeping with this study, the use of mechanical ventilation in only one-third of the patients could be seen as a marker of early ICU admission as much as an indirect sign that life sustaining therapies had been withheld in some patients. Future studies should also inform on the level of care in each treatment groups, showing the proportion of patients not receiving chemotherapy or mechanical ventilation when those treatments were needed.

With the ageing population and the increased number of fit elderly patients receiving intensive chemotherapy, targeted treatments or cell therapy, critical care services will also be increasingly sought for new patient populations, putting forefront frailty and the risk for acute organ dysfunction. Here again, working together with hematologists to assess organ fitness and help recognizing those frail patients likely to benefit from advanced treatments will substantially change the typology of critically ill hematology patients.

In summary, critical care services need to be preempted for patients with hematological malignancies. Patients with aggressive malignancies such as high-grade lymphoma or acute myeloid or lymphocytic leukemia, and those with comorbidities, are high users of ICU resources. These results strongly suggest that ICUs should be designed and scaled based on the number of hematology patients. Future studies should update these findings by taking into account the number of patients who could have benefited from ICU management but were not admitted because of the lack of bed or the use of self-restrictions from both hematologists and ICU specialists. Also, qualitative indicators should be used to correlate outcomes with the appropriate use of ICU treatments, with a careful attention to the use of disproportionate allocation of life-sustaining therapies.

\section{Author details}

${ }^{1}$ Critical Care Department, Saint-Louis Hospital, Paris, France. ${ }^{2}$ Hematology Department of the Saint-Louis Hospital, University of Paris, Paris, France. ${ }^{3}$ Department of Intensive Care Medicine, D'or Institute for Research and Education, Rio de Janeiro, Brazil.

\section{Declarations}

Conflicts of interest

Authors declare no conflict of interest in relation with this editorial.

\section{Publisher's Note}

Springer Nature remains neutral with regard to jurisdictional claims in published maps and institutional affiliations.

Received: 28 August 2021 Accepted: 28 August 2021

Published online: 14 September 2021

\section{References}

1. Zampieri FG, Romano TG, Salluh JIF et al (2021) Trends in clinical profiles, organ support use and outcomes of patients with cancer requiring unplanned ICU admission: a multicenter cohort study. Intensive Care Med 47:170-179. https://doi.org/10.1007/s00134-020-06184-2

2. Vincent J-L, Lefrant J-Y, Kotfis K et al (2018) Comparison of European ICU patients in 2012 (ICON) versus 2002 (SOAP). Intensive Care Med 44:337-344. https://doi.org/10.1007/s00134-017-5043-2

3. Azoulay E, Pickkers P, Soares M et al (2017) Acute hypoxemic respiratory failure in immunocompromised patients: the Efraim multinational prospective cohort study. Intensive Care Med. https://doi.org/10.1007/ s00134-017-4947-1

4. Azoulay E, Mokart D, Kouatchet A et al (2019) Acute respiratory failure in immunocompromised adults. Lancet Respir Med 7:173-186. https://doi. org/10.1016/S2213-2600(18)30345-X

5. Zafrani L, Canet E, Darmon M (2019) Understanding tumor lysis syndrome. Intensive Care Med 45:1608-1611. https://doi.org/10.1007/ s00134-019-05768-x

6. Azoulay E, Russell L, Van de Louw A et al (2020) Diagnosis of severe respiratory infections in immunocompromised patients. Intensive Care Med 46:298-314. https://doi.org/10.1007/s00134-019-05906-5 
7. Asdahl PH, Christensen S, Kjærsgaard A et al (2020) One-year mortality among non-surgical patients with hematological malignancies admitted to the intensive care unit: a Danish nationwide population-based cohort study. Intensive Care Med 46:756-765. https://doi.org/10.1007/ s00134-019-05918-1

8. Azoulay E, Lemiale V, Mourvillier B et al (2018) Management and outcomes of acute respiratory distress syndrome patients with and without comorbid conditions. Intensive Care Med 44:1050-1060. https://doi.org/ 10.1007/s00134-018-5209-6

9. Schellongowski P, Staudinger T, Kundi M et al (2011) Prognostic factors for intensive care unit admission, intensive care outcome, and post-intensive care survival in patients with de novo acute myeloid leukemia: a single center experience. Haematologica 96:231-237. https://doi.org/10.3324/ haematol.2010.031583

10. Wohlfarth P, Staudinger T, Sperr WR et al (2014) Prognostic factors, longterm survival, and outcome of cancer patients receiving chemotherapy in the intensive care unit. Ann Hematol 93:1629-1636. https://doi.org/10. 1007/s00277-014-2141-x

11. Wohlfarth P, Carlström A, Staudinger T et al (2016) Incidence of intensive care unit admission, outcome and post intensive care survival in patients with diffuse large B-cell lymphoma. Leuk Lymphoma 57:1831-1838. https://doi.org/10.3109/10428194.2015.1106537

12. Azoulay $E$, Mokart D, Pène F et al (2013) Outcomes of critically ill patients with hematologic malignancies: prospective multicenter data from France and Belgium-a groupe de recherche respiratoire en réanimation onco-hématologique study. J Clin Oncol 31:2810-2818. https://doi.org/ $10.1200 / J C O .2012 .47 .2365$

13. van Vliet M, van den Boogaard M, Donnelly JP et al (2014) Long-term health related quality of life following intensive care during treatment for haematological malignancies. PLoS ONE 9:e87779. https://doi.org/10. 1371/journal.pone.0087779

14. Ferreyro B, Scales DC, Wunsch $\mathrm{H}$ et al (2021) Critical illness in patients with hematologic malignancy: a population-based cohort study. Intensive Care Med. https://doi.org/10.1007/s00134-021-06502-2

15. Moreau A-S, Lengline E, Seguin A et al (2014) Respiratory events at the earliest phase of acute myeloid leukemia. Leuk Lymphoma 55:2556-2563. https://doi.org/10.3109/10428194.2014.887709

16. Stahl M, Shallis RM, Wei W et al (2020) Management of hyperleukocytosis and impact of leukapheresis among patients with acute myeloid leukemia (AML) on short- and long-term clinical outcomes: a large, retrospective, multicenter, international study. Leukemia 34:3149-3160. https://doi. org/10.1038/s41375-020-0783-3

17. Azoulay E, Pène F, Darmon M et al (2015) Managing critically III hematology patients: time to think differently. Blood Rev 29:359-367. https://doi. org/10.1016/j.blre.2015.04.002

18. Lengliné $E$, Raffoux $E$, Lemiale $V$ et al (2012) Intensive care unit management of patients with newly diagnosed acute myeloid leukemia with no organ failure. Leuk Lymphoma 53:1352-1359. https://doi.org/10.3109/ 10428194.2011.649752 